\title{
NOTE ON A THEOREM OF NEHARI
}

\section{R. M. MORONEY}

In a recent paper [1] Nehari investigated the oscillation of solutions $y(x)$ of

$$
y^{\prime \prime}+y F\left(y^{2}, x\right)=0
$$

under the following conditions on the function $F(t, x)$ :

(2a) $F(t, x)$ is continuous in $(t, x)$ on $\{(t, x): 0 \leqq t<\infty, 0<x<\infty\}$.

$$
F(t, x)>0 \text { for } t>0, x>0 \text {. }
$$

For fixed positive $x$ and some $\epsilon>0$

$$
t_{2}^{-\epsilon} F\left(t_{2}, x\right)>\bar{t}_{1}^{-\epsilon} F\left(t_{1}, x\right) \quad\left(0 \leqq t_{1}<t_{2}<\infty\right) .
$$

In the course of this investigation the question arose whether a $C^{2}$ solution of (1), with $F$ subject to (2), is uniquely determined by the conditions

$$
y(a)=y^{\prime}(b)=0, \quad y(x)>0 \quad \text { for } x \in(a, b] .
$$

Nehari conjectured that this solution is unique under an additional condition on the behavior of $F(t, x)$ as a function of $x$. The purpose of this note is to show that such is the case if $F(t, x)$ satisfies the following condition:

(2d) For each fixed positive $\rho$ and $0 \leqq x_{1}<x_{2}<\infty, F\left(\rho, x_{2}\right) \geqq F\left(\rho, x_{1}\right)$.

We formulate this as a theorem:

Theorem. In (1), let $F(t, x)$ satisfy hypotheses (2a) to (2d). Then for each pair $(a, b), 0 \leqq a<b<\infty$, there exists a unique solution $y(x)$ of (1) on $[a, b]$ satisfying $(\mathrm{C})$ and having two continuous derivatives on $[a, b]$.

Lemma 1. Let $y_{1}(x)$ and $y_{2}(x)$ be two $C^{2}$ solutions of (1) on some interval $[a, c)$ such that

(a) $y_{1}(a)=y_{2}(a)=0, y_{1}^{\prime}(a)=y_{2}^{\prime}(a)>0$.

(b) $y_{i}(x)>0$ and $y_{i}^{\prime}(x)>0$ for $x$ in $(a, c)$.

Then $y_{1}(x)=y_{2}(x)$ on $a \leqq x<c$.

Proof. Suppose $y_{2}(\tilde{x})>y_{1}(\tilde{x})$ for some $\tilde{x}$ in $(a, c)$. Then by the meanvalue theorem there exists an $x_{2}$ in $(a, \tilde{x})$ such that $y_{2}^{\prime}\left(x_{2}\right)>y_{1}^{\prime}\left(x_{2}\right)$ and by a second application an $x_{3}$ in $\left(a, x_{2}\right)$ such that $y_{2}^{\prime \prime}\left(x_{3}\right)>y_{1}^{\prime \prime}\left(x_{3}\right)$. Because of (2b) and the form of (1), however, this implies $y_{2}\left(x_{8}\right)$ $<y_{1}\left(x_{3}\right)$.

Received by the editors April 6, 1961. 
By repeating the foregoing argument one sees that if $y_{2}(x)$ and $y_{1}(x)$ differ at any point of $(a, c)$ a situation as in Figure 1 must arise,

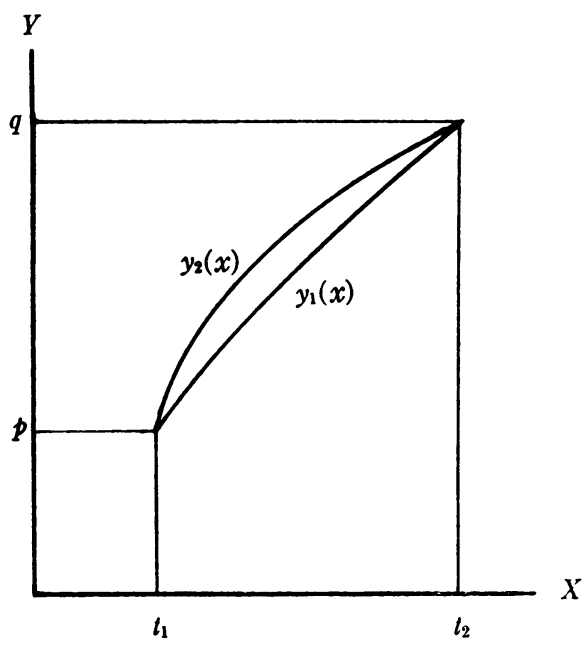

FigURe 1

that is there will exist an $x$ interval $\left[t_{1}, t_{2}\right] \subset(a, c)$ such that $y_{1}\left(t_{i}\right)$ $=y_{2}\left(t_{i}\right), y_{2}(x)>y_{1}(x)$ on $t_{1}<x<t_{2}$, and $y_{2}^{\prime}\left(t_{1}\right)>y_{1}^{\prime}\left(t_{1}\right), y_{2}^{\prime}\left(t_{2}\right)<y_{1}^{\prime}\left(t_{2}\right)$. We now show that this is impossible.

By the continuity of $\left(y_{2}-y_{1}\right)^{\prime}$ as a function of $y$, there will exist $t_{3}$ and $t_{4}$ such that $y_{2}\left(t_{3}\right)=y_{1}\left(t_{4}\right)=r, y_{2}^{\prime}\left(t_{3}\right)=y_{1}^{\prime}\left(t_{4}\right)=z, t_{3}<t_{4}$ (see Figure 2).

By (1), however, on $t_{1} \leqq x \leqq t_{2}$

(3) $y_{2}(x)=y_{2}\left(t_{1}\right)+\left(x-t_{1}\right) y_{2}^{\prime}(x)+\int_{t_{1}}^{x}\left(s-t_{1}\right) y_{2}(s) F\left(y_{2}^{2}(s), s\right) d s$,

(4) $y_{1}(x)=y_{1}\left(t_{1}\right)+\left(x-t_{1}\right) y_{1}^{\prime}(x)+\int_{t_{1}}^{x}\left(s-t_{1}\right) y_{1}(s) F\left(y_{1}^{2}(s), s\right) d s$,

as is easily seen by differentiating. Using (3) at $t_{3}$ and (4) at $t_{4}$ and subtracting gives

$$
0=\left(t_{3}-t_{4}\right) z+\int_{t_{1}}^{t_{3}}\left(s-t_{1}\right) y_{2}(s) F\left(y_{2}^{2}(s), s\right) d s
$$

$$
-\int_{t_{1}}^{t_{4}}\left(s-t_{1}\right) y_{1}(s) F\left(y_{1}^{2}(s), s\right) d s
$$




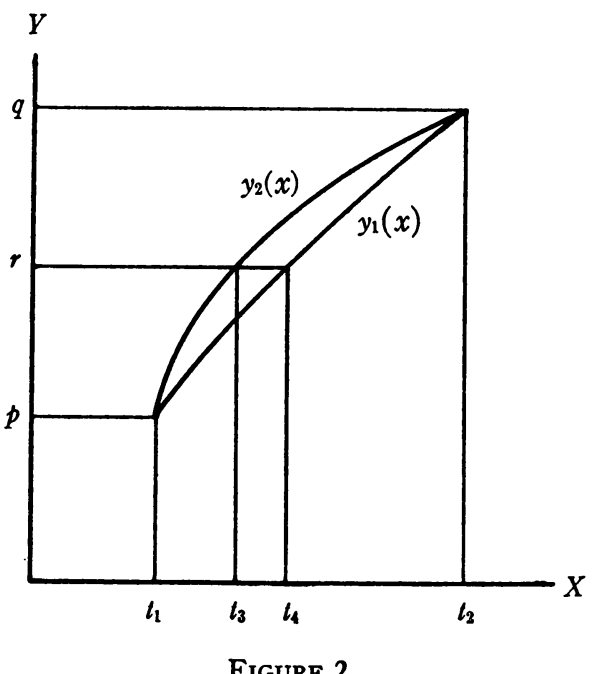

In the first integral make the change of variable $\lambda=y_{2}(s)$, and in the second $\lambda=y_{1}(s)$ :

$$
0=\left(t_{3}-t_{4}\right) z+\int_{p}^{r}\left(s_{2}-t_{1}\right)(\lambda) \frac{\lambda}{\lambda_{\xi}(\lambda)} F\left(\lambda^{2}, s_{2}(\lambda)\right) d \lambda
$$

$$
-\int_{p}^{r}\left(s_{1}-t_{1}\right)(\lambda) \frac{\lambda}{y_{1}^{\prime}(\lambda)} F\left(\lambda^{2}, s_{1}(\lambda)\right) d \lambda .
$$

Since for each $\lambda$ in $[p, r]\left(s_{1}-t_{1}\right)(\lambda) \geqq\left(s_{2}-t_{1}\right)(\lambda)$ and $y_{1}^{\prime}(\lambda) \leqq y_{2}^{\prime}(\lambda)$, while by hypothesis (2d) $F\left(\lambda^{2}, s_{2}(\lambda)\right) \leqq F\left(\lambda^{2}, s_{1}(\lambda)\right)$, the difference between the integrals in (6) is negative. So is the first term, however, so the right side can not be zero.

LEMMA 2. Let $y_{2}(x)$ and $y_{1}(x)$ be solutions of (1) on $[a, b]$ such that $(i=1,2)$

$$
\begin{array}{rlr}
y_{i} & \in C^{2}[a, b] \\
y_{i}(a) & =y_{i}^{\prime}(b)=0 \\
y_{i}(x) & >0 &
\end{array}
$$

Then $y_{2}^{\prime}(a)=y_{1}^{\prime}(a)$.

Proof. Suppose $y_{2}^{\prime}(a)>y_{1}^{\prime}(a)$, and consider the function $\left(y_{2} y_{1}^{\prime}-y_{1} y_{2}^{\prime}\right)(x)$. We have

$$
\left(y_{2} y_{1}^{\prime}-y_{1} y_{2}^{\prime}\right)^{\prime}=y_{2} y_{1}\left[F\left(y_{2}^{2}, x\right)-F\left(y_{1}^{2}, x\right)\right]
$$




$$
\left(y_{2} y_{1}^{\prime}-y_{1} y_{2}^{\prime}\right)(x)=\int_{a}^{x} y_{2}(s) y_{1}(s)\left[F\left(y_{2}^{2}(s), s\right)-F\left(y_{1}^{2}, s\right)\right] d s .
$$

By hypothesis $\left(y_{2} y_{1}^{\prime}-y_{1} y_{2}^{\prime}\right)(b)=0$. The integrand in $(7)$ is positive, however, as long as $y_{2}(s)>y_{1}(s)$ and in particular on some interval $(a, \alpha)$ - because $\left(y_{2}-y_{1}\right)^{\prime}(a)>0$. In fact, $\alpha$ may be taken as $b$ because the same argument as in Lemma 1 shows that the graphs of $y_{2}(x)$ and $y_{1}(x)$ can not intersect on $(a, b)$. Thus the right side of $(7)$ does not tend to zero as $x \rightarrow b-$.I

PROOF OF THE THEOREM. The existence of at least one solution of $(1)+(C)$ has been proved by Nehari [1, Theorem IV]. By the preceding lemmas there is at most one such solution.

\title{
REFERENCE
}

1. Zeev Nehari, On a class of nonlinear second-order differential equations, Trans. Amer. Math. Soc. 95 (1960), 101-123.

Massachusetts Institute of Technology

\section{ON THE MEASURABILITY OF FUNCTIONS IN TWO VARIABLES}

\author{
MARK MAHOWALD ${ }^{1}$
}

Let $(X, \mu)$ and $(Y, \nu)$ be two compact spaces having regular Borel measures defined on them. By a measurable modification $\tilde{f}(x, y)$ of a function $f(x, y)$ we mean a function measurable in both variables together and for which $\tilde{f}(x \cdot)=f(x \cdot)$ almost everywhere $[\nu]$ for every $x$. The purpose of this note is to prove the following theorem.

THEOREM. If $Y$ is metric and if $f(x, y)$ has a measurable modification and $f(x \cdot)$ is continuous for almost all $x$, then $f(x, y)$ is measurable in both variables together.

This theorem was discovered in an effort to prove that the Nelson canonical version [2] is measurable if it has a measurable modification. The theorem would prove this result except for the restriction that $Y$ be metric.

Received by the editors December 30, 1960 and, in revised form, April 14, 1960.

${ }^{1}$ Research supported by the United States Air Force Office of Scientific Research of the Air Research and Development Command under Contract No. AF 49(638)-265. Reproduction in whole or part is permitted for any purpose of the United States Government. 